\title{
BMJ Open Effect of joint transition visits on quality of life in adolescents with inflammatory bowel diseases: a protocol for a prospective, randomised, multicentre, controlled trial (TRANS- IBD)
}

\author{
Adrienn Erős, ${ }^{1,2,3}$ Dóra Dohos, ${ }^{1,3}$ Gábor Veres, ${ }^{4}$ András Tárnok, ${ }^{5}$ Áron Vincze,${ }^{6}$ \\ Alexandra Tészás, ${ }^{5}$ Noémi Zádori, ${ }^{1,3}$ Noémi Gede, ${ }^{1,3}$ Péter Hegyi, ${ }^{1,3,6,7}$ \\ Patrícia Sarlós (10) ${ }^{1,6}$
}

To cite: Erős A, Dohos D, Veres G, et al. Effect of joint transition visits on quality of life in adolescents with inflammatory bowel diseases: a protocol for a prospective, randomised, multicentre, controlled trial (TRANS-IBD). BMJ Open 2020;10:e038410. doi:10.1136/ bmjopen-2020-038410

- Prepublication history for this paper is available online. To view these files, please visit the journal online (http://dx.doi. org/10.1136/bmjopen-2020038410).

Received 09 March 2020 Revised 14 August 2020 Accepted 20 August 2020

Check for updates

(C) Author(s) (or their employer(s)) 2020. Re-use permitted under CC BY-NC. No commercial re-use. See rights and permissions. Published by BMJ.

For numbered affiliations see end of article.

Correspondence to Dr Patrícia Sarlós; sarlosp@gmail.com

\begin{abstract}
Introduction Inflammatory bowel diseases (IBD) are among the most common chronic illnesses diagnosed in childhood. Transition from paediatric to adult care is a crucial phase. The implementation of joint visits during the transition period in IBD is widely recommended, however, strong evidence supporting their benefit is still missing. In this trial, we aim to prove the superiority of joint visits compared with usual care in improving transition outcomes of adolescents with IBD.

Methods and analysis This is a randomised controlled two-arm multicentre trial. A minimum of 160 adolescents with IBD aged between 16.75 and 17 years will be recruited from Hungarian tertiary IBD centres. After randomisation, eligible subjects in the intervention arm attend a total of four joint visits with adult and paediatric gastroenterologist between the ages of 17 and 18. In the control arm, adolescents meet only the paediatric gastroenterologist, but there is a balanced consultation between the two gastroenterologist regarding the patient's treatment plan. Patients in both groups receive the same training and education, the only determinative difference between the two arms is the presence of the adult gastroenterologist at the joint visits. Data will be collected at inclusion, at transfer and 12 months post-transfer. Primary outcome is the change in health-related quality of life measured with the IMPACT-III questionnaire at 1 year after transfer. Secondary outcomes include the number of patients not lost to follow-up, healthcare utilisation, disease activity, medication adherence, self-efficacy, transition readiness and patient's satisfaction. To compare the results of the two patient groups, two-sample T-test and Mann-Whitney test will be applied.

Ethics and dissemination The Scientific and Research Ethics Committee of the Hungarian Medical Research Council approved this study (50457-2/2019/EKU). Findings will be disseminated at conferences and in medical journals.

Trial registration number NCT04290156.
\end{abstract}

Strengths and limitations of this study

- This is the first randomised controlled trial which aims to provide strong scientific evidence on the superiority of joint visits compared with standard transitional care in IBD.

- As the trial investigates the effect of joint transition visits on several individual and healthcare outcomes, its results will help to better define the success of the transition process and to determine the long-term influence of joint transition visits in IBD.

- Due to the nature of the study, double blinding is not applicable, as the gastroenterologists, the medical staff and the participants cannot be blinded.

- After 18 years of age, young adults tend to move to other cities for further education or employment, which involves the possibility of losing patients during the follow-up period.

\section{INTRODUCTION}

Due to the increasing number of children diagnosed with chronic diseases, there is a growing need for reliable, evidence-based guidelines dealing with transitional care. ${ }^{1}$ The currently available guidelines on providing transitional care in inflammatory bowel disease (IBD) recommend the implementation of joint visits. ${ }^{23}$ However, these recommendations are mainly based on clinical experiences, rather than strong scientific evidence. In order to be able to assure a healthcare service of the highest quality for adolescent patients with IBD, further comparative investigation of the transition process is needed.

\section{Transition outcomes}

Recently, several international Delphi studies were conducted aiming to identify outcomes 


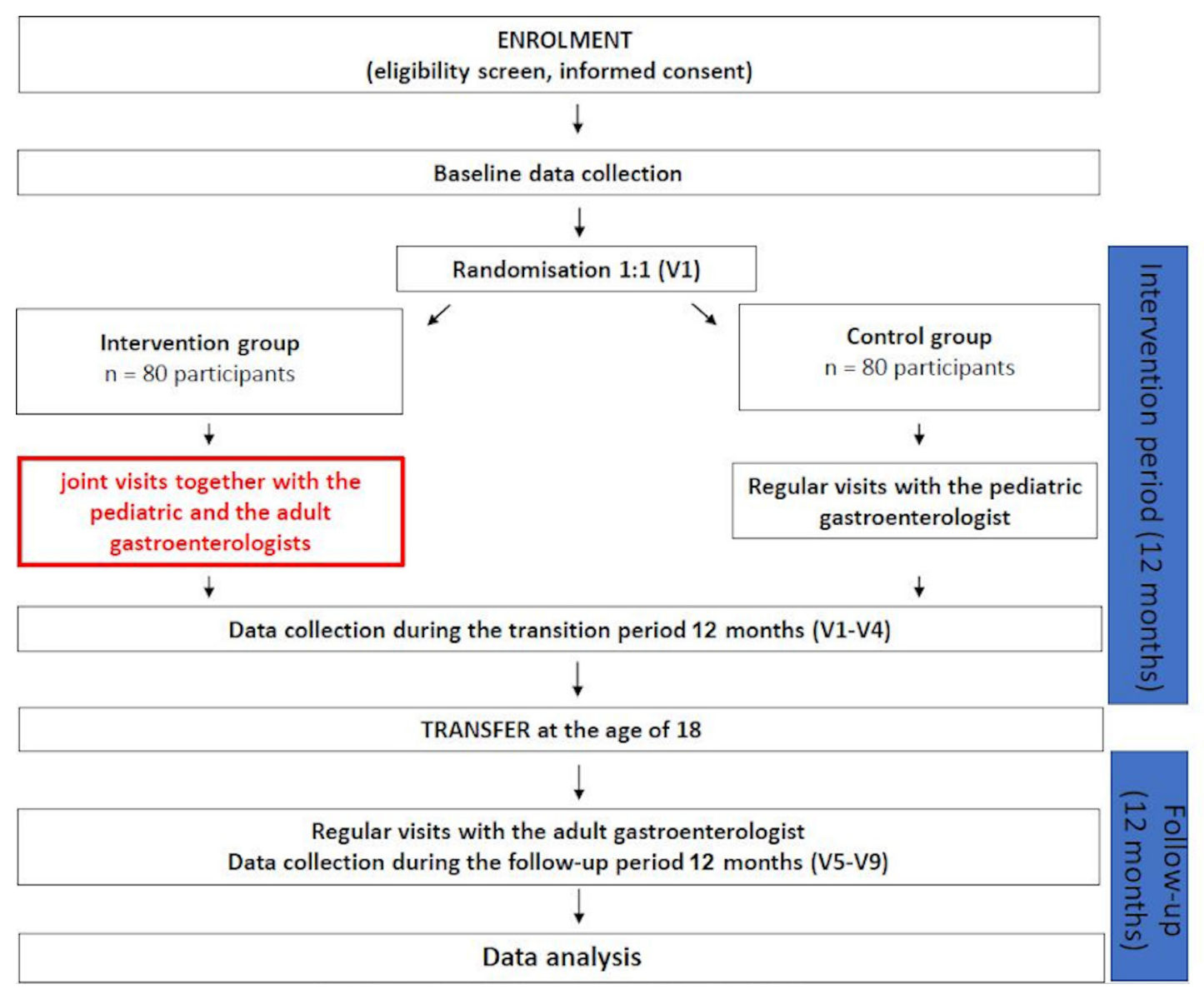

Figure 1 Flow chart of the study design.

of transition. According to the data of non-disease-specific studies, patients not lost to follow-up, health-related quality of life (HRQoL), self-management and disease-specific knowledge are among the most important indicators of a successful transition. ${ }^{45}$ According to a multinational IBD-specific Delphi study, individual outcomes during transition were considered more important than diseaserelated items. ${ }^{6}$ As transitional care is a complex process, it is difficult to define and prioritise endpoints. Based on the most up-to date observations, individual, healthcare service and social outcomes should be taken equally into account as composite endpoints of a transition process in IBD. ${ }^{4-6}$

\section{Transitional interventions}

In a recent systematic review, several different structured transition interventions were identified aiming to improve transition-related outcomes. ${ }^{7}$ Joint visits were the most frequently applied interventions, which were held under different circumstances and were organised by multidisciplinary groups of different compositions all around the world. ${ }^{8-12}$ Non-randomised studies with low sample sizes and selection bias showed that joint visits may improve quality of life, medical adherence, patients' satisfaction and able to optimise medical visit attendance rates. ${ }^{8}{ }^{13-17}$ However, patient education programmes and the coordinating role of IBD nurses are also considered to be beneficial in structured transition interventions. ${ }^{18-23}$

\section{Need for a trial}

Since the quality of evidence proved to be very low for each transitional outcome, further trials are needed to determine the active ingredient and the long-term impact of these interventions. ${ }^{7}$ Joint visits were mainly held in the presence of a paediatric and an adult gastroenterologist (PGE and AGE, respectively), hence the real impact of the personal attendance of the AGE on the whole transition process is questionable. Moreover, the cost-effectiveness of transition programmes can be questioned. This randomised controlled trial (RCT) aims to establish evidence on whether joint transition visits for adolescents with IBD are superior to standard care.

\section{Aim and hypothesis}

This RCT aims to establish whether joint visits are superior to standard transitional care at improving the HRQoL for adolescents with IBD. We hypothesise that the personal interaction between the AGE and the adolescent during the transition period can significantly improve both individual (HRQoL, medication adherence, self-efficacy, transition readiness, patient satisfaction) and healthcare outcomes (medical visit attendance, avoidance of unnecessary hospitalisations). 


\section{METHODS AND DESIGN}

\section{Trial organisation, committees and boards}

TRANS-IBD is designed and coordinated by the Centre for Translational Medicine (http://www.tm-pte.org/; Medical School, University of Pécs), in which several clinical trials have already been initiated (GOULASH, EMILY and ELEFANT ${ }^{24-26}$ on the field of gastroenterology aiming to improve the quality of healthcare). The study protocol was planned in accordance with the SPIRIT 2013 Statement (online supplemental material 1). ${ }^{27}$

\section{Steering committee}

The Steering committee (SC) will be led by PS (gastroenterologist). The members will be $\mathrm{AE}$ and $\mathrm{DD}$ (medical doctors, full-time employees on the project), PH (gastroenterologist), ÁV (gastroenterologist) and ATá (PGE). The SC will make decisions on all relevant questions concerning the participation and the dropouts during the study.

\section{General study overview}

This study is a prospective, multicentre RCT. The patient recruitment is performed in those gastroenterology centres of Hungary, where both paediatric and adult IBD clinics are available. Eligible adolescents, who have agreed to participate in our RCT, are randomised into two arms (figure 1). Patients in the intervention arm receive usual medical care plus a transition intervention for 1 year consisting of four joint sessions of experts including PGE and AGE. Transitional care with joint visits is not standard in the Hungarian medical care system. In the control arm, which corresponds to the standard of care in Hungary, participants follow their usual medical care without the presence of AGE at outpatient consultations. The intervention period lasts between the ages of 17 and 18 (visits $1-4$; V1-V4); at the age of 18 transfer to adult gastroenterology is obligatory. After 1-year follow-up period at the adult healthcare system (visits 5-9; V5-V9), the trial ends at the age of 19 of the adolescents. During the study period, patients are not allowed to change study groups. In order to improve participants' adherence to the study protocol, the next appointment of each visit is arranged in advance and rescheduled visits are also accepted.

Questionnaires are filled out at baseline, at the time of transfer to adult healthcare and at 1-year post-transfer. The monitoring of physical development, disease activity, healthcare utilisation and adverse events is continuous during the study. Patients in the intervention and control groups are treated by the same physician and under the same conditions in each recruiting centre. Adolescents in both study groups are transferred to the same AGE. Modifications of therapy are easy to track because names of currently taken IBD-related drugs and side effects are recorded on the case report form, which is completed at each clinical visit during the study. To promote participant retention in the study and complete follow-up, patients planning to move to other city after the age of 18 are offered to be followed up by another
AGE working in the destination city (who is also the participant of TRANS-IBD study).

\section{Participating centres}

Study participants are recruited from six tertiary paediatric care centres in Hungary (University of Pécs, Debrecen, Szeged, Central Hospital of Borsod-Abaúj-Zemplén County, Semmelweis University Budapest and Heim Pál Children's Hospital). The adult gastroenterology sites are the corresponding tertiary centres in Hungary, namely Pécs, Debrecen, Szeged, Miskolc and two additional centres from Budapest. The principal investigators at the various sites are members of the Hungarian Pediatric Gastroenterology Society and the Hungarian Society of Gastroenterology. The local investigators introduce the study to the adolescents and parents and provide a letter of information. As our study is an openlabel trial, we aim to recruit further Hungarian and CentralEastern European centres for participation. We welcome the joining of those IBD centres which can recruit eligible adolescent participants and can provide the continuous participation of a PGE and an AGE at the joint visits. Centres with the intention of joining need to send a letter of intent to the corresponding author by email.

\section{Inclusion and exclusion criteria}

The inclusion criteria are: (1) established IBD diagnosis based on the modified 'Porto Criteria' at least 6 months prior to enrolment (date of the diagnostic endoscopy) ${ }^{28}$; (2) any form of IBD (including Crohn's disease or ulcerative colitis) regardless of disease activity and treatment; (3) patient aged between 16.75 and 17 years at allocation; (4) at least one visit attendance at the PGE in the year prior to enrolment (aiming to minimise non-adherence with the intervention); (5) signed written informed consent from the legal guardian and informed assent from the patients.

The exclusion criteria are: (1) diagnosis of unclassified IBD; (2) pregnancy; (3) medically certified developmental or intellectual disabilities (when it is expected that the patient is unable to fill the questionnaires); (4) history of cancer or active cancer treatment; (5) body mass index $\geq 40$, to eliminate the impact of potential comorbidities on our primary outcome; (6) concomitant participation in another interventional clinical trial; (7) conditions when follow-up cannot be fulfilled (eg, plan for studying or working abroad after the age of 18).

\section{Patient and public involvement}

During the trial planning stage, adolescents' preferences were taken into account. To involve patients in the development of study protocol, we invited five adolescents with IBD to revise two version of the study protocol. In the first version, the intervention period lasted for 2years between the ages of 16 and 18 with joint transition visits in every sixth months. In the second version, the intervention period was planned for only 1 year just before the transfer to the adult healthcare with joint visits held in every third month. The invited patients assessed the burden of the intervention in case of both study versions and reported greater preferences for the 
second version of the protocol. The results of the study will be disseminated to the participants through the AGEs involved in the study. Herewith, we would like to thank the adolescents who were actively involved in the development of our study protocol.

\section{Recruitment}

Eligible adolescents (aged 16.75-17 years) treated with IBD and their parents are informed about the possibility to participate in the study. After the PGE has expounded the main points of the trial, adolescents and their legal guardians should confirm their intention to participate by signing the informed consents. The flow chart of the study is summarised in figure 1.

\section{Baseline assessment}

At the first transition visit, right before randomisation, selfreported background information questionnaire is obtained from patients, comprising sociodemographic variables such as age, gender, ethnicity, birth, level of education, number of siblings, living situation, family structure, parent's highest level of education and smoking habits. Medical history (date of diagnosis, Paris classification at diagnosis, ${ }^{29}$ previous abdominal surgeries, medications and adverse events), comorbidities, body weight, height, concomitant medications, disease activity (assessed with adult and paediatric disease activity indices and stool calprotectin), ${ }^{30-33}$ standard laboratory parameters (haematology, biochemistry and inflammatory markers) and utilisation of healthcare services (in the previous 3 months) are also assessed. Additionally, the questionnaires measuring transition-related outcomes are also filled out by the participants (listed among the primary and secondary outcomes).

\section{Randomisation and blinding}

After obtaining the informed consents at visit 1 (V1), in each participating centre, patients are block-randomised with a random variable block sizes of two, four and six to the intervention group or the comparison group beside a 1:1 allocation ratio. A randomisation list will be generated for each participating centre by the biostatistician group of the Centre for Translational Medicine with the application of a computer program. Sequentially numbered, sealed envelopes will contain the assigned treatment group for the next participant.

Due to the nature of the study, the blinding of the participants and personnel (PGE, AGE, medical staff) is not possible, however, the blinding of the data managers and statisticians will be secured.

\section{Balanced consultation}

In the strict sense, our study involves two different interactions: (1) the interaction between the PGE and the AGE and (2) the interaction between the AGE and the adolescent. The aim of this study is to assess the effect of the interaction between the AGE and the adolescent. Balanced consultations are carried out to eliminate the bias caused by the physicianphysician interaction, which are not currently part of the standard clinical care in Hungary. Balanced consultations are held in both study groups, before each visit during the intervention period, and provide an opportunity for the AGE to make recommendations on the patient's medical history and the actual treatment options. The two gastroenterologists should also have a discussion regarding the information package that is provided for the participant in connection with the transition process (online supplementary material 2). In summary, a balanced consultation is a clinical review between the two gastroenterologists without the presence of the adolescents involved.

\section{Elimination of other confounding factors}

Beside balanced consultation, we aim to minimise all the other potential confounding factors. Regarding treatment plan, the guidelines of the European Crohn's and Colitis Organisation are followed in both groups. ${ }^{34-36}$ All patients access services that address their healthcare needs. If indicated, dietitian, psychologist and surgeon are available for every participant.

Regardless of the study group, patient education comprises information about the transition-related topics controlled by the predefined 'TRANS-IBD Information sheets 1-4' (online supplementary material 2). In both study groups, the transfer from paediatric to adult care includes a scheduled appointment at the previously assigned AGE and a letter summarising the patient's medical history.

\section{Intervention (treatment) group}

In our clinical trial, the structured transition intervention is the application of joint transition visits with the simultaneous participation of the PGE and the AGE. In order to maximise the effect of the intervention, the implementation of four joint visits during the 1-year intervention period was chosen. The four face-to-face sessions take place every third month between the ages 17 and 18 and are localised at the paediatric outpatient clinic (=transition clinic). Each joint visit lasts at least for $20 \mathrm{~min}$ in length, although in case of complex medical history, there is no restriction with respect to the length of the visit. Joint transition visits 1, 2 and 3 (V1-V3) are led by the PGE but visit 4 (V4) is led by the AGE. During joint transition visits, transition and age-related topics are discussed (online supplementary material 2).

\section{Control group (usual care)}

Patients in the control group are given standard of care and visit their PGE every 3 months between their 17 and 18 years of age. As patients in the control group do not participate on joint visits, they have no contact with the AGE through the intervention period. During medical visits with the PGE, transition and age-related topics are discussed likewise in the intervention group (online supplementary material 2). Before each visit, balanced consultation is performed about the adolescent's treatment plan to ensure equality.

\section{Outcome measures}

Careful selection of outcome measures for adolescents is important to ensure reliability, objectivity and feasibility. Outcome measures of our study were selected based on 
Table 1 Schedule of enrolment, interventions and assessments

\begin{tabular}{|c|c|c|c|c|c|c|}
\hline Study-related procedures & Enrolment & Intervention period & & Follow-up & & Close-out \\
\hline $\begin{array}{l}\text { Visits } \\
\text { Patient's age }\end{array}$ & $\begin{array}{c}\text { Visit } 0 \\
16.75-17 \text { years }\end{array}$ & $\begin{array}{c}\text { Visit } 1 \\
17 \text { years } \pm 30 \text { days }\end{array}$ & Visit 2-3-4* & Visit 5 & Visit 6-7-8* & $\begin{array}{c}\text { Visit } 9 \\
19 \text { years } \pm 30 \text { days }\end{array}$ \\
\hline
\end{tabular}

Enrolment

\begin{tabular}{|c|c|c|c|c|c|c|}
\hline Eligibility screen & $x$ & & & & & \\
\hline Informed consent & $x$ & & & & & \\
\hline Allocation & $x$ & & & & & \\
\hline Intervention group & PGE & PGE+AGE & $\mathrm{PGE}+\mathrm{AGE}$ & AGE & AGE & AGE \\
\hline Control group & PGE & PGE & PGE & AGE & AGE & AGE \\
\hline Medical history & & $\mathrm{x}$ & & & & \\
\hline Physical examination† & & $x$ & $x$ & $x$ & $x$ & $x$ \\
\hline Body height and weight & & $x$ & $x$ & $x$ & $x$ & $x$ \\
\hline Actual medications & & $x$ & $x$ & $x$ & $x$ & $x$ \\
\hline Healthcare utilisation $\ddagger$ & & $x$ & $x$ & $x$ & $x$ & $x$ \\
\hline Serum sample§ & & $x$ & $x$ & $x$ & $x$ & $x$ \\
\hline Stool samplef & & $x$ & & $x$ & & $x$ \\
\hline \multicolumn{7}{|l|}{ Questionnaires } \\
\hline Self-efficacy (IBDSES-A) & & $x$ & & $x$ & & $x$ \\
\hline Transition readiness (TRAQ) & & $x$ & & $x$ & & $x$ \\
\hline $\begin{array}{l}\text { Transition readiness (STARx- } \\
\text { adolescent version) }\end{array}$ & & $x$ & & $x$ & & $x$ \\
\hline $\begin{array}{l}\text { Transition readiness (STARX- } \\
\text { parent version) }\end{array}$ & & $x$ & & $x$ & & $x$ \\
\hline
\end{tabular}

$\mathrm{X}=$ scheduled measurements.

${ }^{*}$ During the study, visits are held in every three months $( \pm 30$ days).

†First visit: complete physical examination; further visits: symptom-oriented physical.

fIBD-related healthcare utilisation (considering the last 3 months): 1. number of unscheduled visits at the caregiver gastroenterologist; 2. number of unplanned/unscheduled visits at the emergency department; 3 . number of scheduled and urgent imaging; 4 . number of scheduled and urgent endoscopies; 5 . number and type of surgical interventions; 6. number of IBD-related hospital admissions; 7 . length of hospitalisation.

$\S$ Determination of laboratory parameters (haematology, biochemistry, inflammatory markers).

qDetermination of stool calprotectin.

${ }^{* *} \mathrm{HRQ} \mathrm{R}$ will be measured at visit 7 .

AGE, medical visit with the adult gastroenterologist; CDAl, Crohn Disease Activity Index; HRQoL, health-related quality of life; IBD, inflammatory bowel disease; IBDSES-A, IBD Self-Efficacy Scale for adolescents and young adults; MARS-5, Medical Adherence Report scale; PCDAI, Paediatric Crohn's Disease Activity Index; PGE, medical visit with the pediatric gastroenterologist; PGE + AGE, joint visit with the attendance of both pediatric and adult gastroenterologists; PUCAI, Paediatric Ulcerative Colitis Activity Index; STARx-adolescent, STARx Transition Readiness Questionnaire (Adolescent Version); STARx-parent, STARx Transition Readiness Questionnaire (Parent Version); TRAQ, Transition Readiness Assessment Questionnaire.

three international consensus publications and two systematic reviews. ${ }^{4-7} 37$ In the development phase, final items were chosen with the stakeholder reference group through discussion. In table 1 , an overview of all variables and instruments at each time point is provided. Primary and secondary outcomes are assessed at baseline, at the end of the intervention period and at the end of the follow-up period. The questionnaires used in our study are validated and most of them are disease specific. Forward-backward translation of the questionnaires was performed by two independent native Hungarian speakers with excellent knowledge of English. The IMPACT-III questionnaire is a valid and reliable 
questionnaire for assessing HRQoL of children with IBD, which has been already adapted in Hungary. ${ }^{38}$ The further questionnaires used for the assessment of the secondary outcomes will be adapted as part of the study.

\section{Primary outcome}

The primary outcome of our trial is the change in patientreported HRQoL 1 year after transfer focusing on patients' subjective evaluation about the impact of the disease on different health dimensions (including physical, emotional, social and cognitive functions). ${ }^{39}$ HRQoL is measured with a validated and IBD-specific QoL questionnaire. ${ }^{40}$ The IMPACT-III questionnaire, which has been already adapted in Hungary, consists of 35 items using a 5-point Likert selfcompleted response scale and asks about the severity and frequency of the following symptoms over the last 2 weeks: bowel symptoms, systemic symptoms, emotional functioning, social functioning, body image and treatments or interventions. ${ }^{38}$ The maximum of 175 points can be achieved. Higher scores indicate better HRQoL. Based on the recommendation of a recently conducted multinational Delphi study on the transition care of adolescent with IBD, HRQoL should be assessed with IMPACT-III questionnaire at 1-year post-transfer. ${ }^{6}$

\section{Secondary outcomes}

The number of patients not lost to follow-up

Patients are not lost to the follow-up if they attend at least three out of the five planned AGE visits during the follow-up period after transfer to adult healthcare. Previously cancelled and then rescheduled AGE visits are acceptable. According to the study protocol, the first AGE visit should be held no later than 3-4 months after transfer.

\section{Healthcare utilisation (measured in every 3 months)}

We are administrating (1) the number of unplanned visits at the caregiver gastroenterologist; (2) the number of unplanned visits at the emergency department; (3) the overall number of scheduled and urgent imaging performed (including abdominal ultrasound, X-ray, MRI, CT scan, other); (4) the overall number of scheduled and urgent endoscopies performed (including gastroduodenoscopy, colonoscopy); (5) the number and type of surgical interventions; (6) the number of IBD-related hospital admissions and (7) the length of disease-related hospitalisation (given in days).

\section{Disease activity}

We will calculate both the paediatric and adult activity indexes at each visit (Paediatric Crohn's Disease Activity Index; Paediatric Ulcerative Colitis Activity Index; Crohn's Disease Activity Index, CDAI, Mayo score and perianal CDAI) and measure the inflammatory laboratory parameters at each visit (eg, C-reactive protein, erythrocyte sedimentation rate and white blood cell); and the level of stool calprotectin once a year. ${ }^{30-3341}$ We will record the number of patients who needed the initiation of corticosteroid treatment and biological treatment and also the number of flare-ups. Flare-ups are defined as clinical symptoms suggesting disease activity, accompanied with biochemical (eg, stool calprotectin, CRP), endoscopic or imaging evidence of inflammation. Intensifying disease symptoms resulting in dose escalation or initiation of a new drug aiming to achieve remission is also considered as flare-ups.

\section{Medication adherence}

Medication adherence is measured with the Medical Adherence Report Scale (MARS-5), which has been already validated in several countries. ${ }^{42-44}$ MARS-5 is a shortened and modified version of the original MARS questionnaire, which consists of five statements with respect to the patient's medical adherence in the last 1 week. ${ }^{45}$ Patients can describe their behaviour on a 5-point Likert response scale ranging from 'always' to 'never' (1-5 points). The lowest total score that can be achieved is 5 (lowest adherence), while the highest is 25 (maximal adherence). Higher scores indicate better medical adherence.

The longitudinal change of patient-reported HRQOL during the trial HRQoL will be measured at baseline, at the beginning (V5), in the middle (V7) and at the end (V9) of the follow-up period.

\section{Self-efficacy}

Self-efficacy is measured with a valid, IBD-specific questionnaire named: IBD Self-Efficacy Scale for adolescents and young adults (IBDSES-A). ${ }^{46-48}$ Through the 13 disease-specific items, IBDSES-A investigates the person's confidence in their ability to manage demands and is predictive of health outcomes in chronic disease such as hospitalisation and health status. Answers can be given on a 5-point Likert response scale in case of every item. The maximum scores range from 21 to 57 (as reverse scoring is used in case of two items). Higher scores indicate higher self-efficacy.

\section{Transition readiness}

Transition readiness is measured with the validated tools of Transition Readiness Questionnaire (STARx) and the Transition Readiness Assessment Questionnaire (TRAQ). ${ }^{49-52}$

STARx consists of three modules (with a total of 18 items), aiming to investigate the sense of responsibility, and the independency of the patients. ${ }^{49}$ Each item can be answered using a 5-point Likert response scale and can be scored between 1 and 5 points. The maximum score is 90 . Since we would like to compare how adolescents themselves and their parents judge participants' transition readiness, STARx is filled out by the participating adolescents and by their legal guardian too. ${ }^{452}$

TRAQ consisting of 20 items and assesses the adolescents' willingness to be involved in or to be completely independent in managing disease-related tasks (eg, taking medications, making appointments, getting medications prescribed).$^{50}$ A 5-point Likert response scale (with scores ranging from 1 to 5 ) is used for answering each question. 
A total of 100 points can be acquired. In case of both tool, higher total scores indicate a higher level of transition readiness.

Although both questionnaires assess adolescents' transition readiness, and some of their questions are almost the same, it should be noted that certain areas of transition readiness occur only in one of the two questionnaires. For example, only TRAQ asks questions about documenting health tasks, while STARx do not ask this topic. ${ }^{4950}$ On the other hand, STARx examines disease-specific knowledge in more detail. ${ }^{49}$

\section{Patient's satisfaction}

Patients' satisfaction is assessed with a validated, IBDspecific tool called CACHE questionnaire, which consists of 31 questions with respect to the attitude of the medical team, the location, accessibility and facilities of the IBD centre. ${ }^{53}$ Questions can be answered with a 5-point Likert response scale. The final score can be given on a scale that ranges from 0 (minimum satisfaction) to 100 (maximum satisfaction). The final total score is calculated with standardisation, using the following formula to determine the points of each individual item: (real score - minimum score $) /($ maximum score - minimum score $) \times 100$.

\section{Data collection and management}

The data collected will be handled and validated by an Independent Data Management Board (IDMB). In addition, IDMB will perform the independent assessment of the trial-related files and activities aiming to ensure the safety of the participants and guarantee the reliability of the collected data. In all cases, the IDMB will perform an audit of the centres wishing to join and will report to the SC.

The data collection is continuous during the intervention and follow-up period. The interventions, the measured variables and the applied instruments with their specified time points are listed for both study groups in table 1. First, data are collected on paper by filling out the case report forms (online supplementary material 3). The visit forms (including the patient's actual symptoms, laboratory parameters, score of activity indices, ongoing therapy, the result of the physical examination and the imaging studies) are filled out by the study nurse. The study nurse is the regular nurse who is involved in the adolescent's healthcare. The questionnaires are filled out also on paper by the participants and parents. Thereafter, the completed visit forms and questionnaires will be entered to a centralised electronic data storage system by administrators. Patients' data are stored anonymously. Paperbased documentations containing personal data can only be accessed by those directly involved in the research.

\section{Adverse events and safety}

Due to the nature of the intervention, the occurrence of intervention-related serious adverse events is not expected. However, adverse events (eg, adverse drug experiences) will be collected from the time of signing the informed consent.

\section{Drop-out}

The collected data will be analysed separately for the intention-to-treat and also for the per-protocol study populations. The intention-to-treat analysis will include the data of all participants who are randomised in the study, even if they violate the protocol or have missing data. The per-protocol analysis will include the data of patients who complete the study according to the requirements of the study protocol. Patients automatically drop-out from the per-protocol analysis if: (1) at least one joint visit is missed; or (2) any of the data considering the primary endpoints cannot be obtained from the participant.

\section{Sample size}

Sample size calculation was based on the article of Cohen et al and was performed by the biostatistician team of the Institute of Translational Medicine. ${ }^{54}$ Based on the available data, we expect nine points difference between the mean IMPACT-III points of the intervention and control group at the end of the study. In order to detect this difference between the two study groups, 160 patients are needed to be recruited (80/group) using a $21 \%$ drop-out rate, $80 \%$ power and $95 \%$ significance level.

\section{Statistical analyses}

The baseline characteristics of the participants will be analysed using descriptive analysis. The data on baseline characteristics and demographic will be described for the overall study population and for the intervention and control groups separately, to reveal any significant difference between the two study populations. In case of continuous variables, mean, median, SD and the quartiles of 25 and 75 will be used to display the results. In case of categorical variable, the results will be described by using absolute or relative frequencies. In case of normal distribution, independent T-test will be used to compare the results of the two study groups, otherwise Mann-Whitney test will be applied. The primary outcome HRQoL will be analysed with analysis of covariance, taking into account disease activity as an influencing factor. For the longitudinal analysis of HRQoL, a mixed model with a proper covariate matrix will be performed. As for the secondary outcomes, the relative risk will be calculated for dichotomous variables and the T-test will be performed for continuous variables.

\section{Interim analyses and premature termination of the study}

Since the recruitment period is expected to be shorter than the follow-up period, no interim analysis will be performed during the study.

\section{Trial duration}

The starting date of the trial is 1 September 2020 and the expected date of finishing the recruitment is 1 September 2024.

\section{Dissemination and publication policy}

Findings will be disseminated at research conferences and in medical journals. Centres can add one or more authors to the authorship list if they: (1) make a substantial contribution 
to the acquisition of the data (recruiting at least 25 participants) and; (2) ensure that questions related to the conduction of the investigation are appropriately investigated and resolved and; (3) take part in the critical review of the draft version of the work and; (4) give their final approval of the version to be published.

\section{Ethics}

The protocol has been approved by the Scientific and Research Ethics Committee of the Medical Research Council (reference number: 50457-2/2019/EKU). Sitespecific approval has been granted by local RECs at all trial sites. The study will be performed in accordance with the Declaration of Helsinki and the principles of International Council for Harmonisation of Technical Requirements for Registration of Pharmaceuticals for Human Use- Good Clinical Practice (ICH-GCP) guidelines. ${ }^{55} 56$

\section{DISCUSSION}

Here we present the protocol of a prospective, randomised, multicentre, controlled trial (TRANS-IBD) aiming to investigate the influence of joint transition visits in IBD. Recent guidelines recommend the performance of joint visits during the transition period, but the strong evidence proving their superiority compared with usual care is still missing. ${ }^{23}$ Our main hypothesis is that the introduction of joint visits with the personal attendance of the AGE will significantly improve the individual HRQoL of the adolescents compared with usual care. Additionally, this structured transition intervention can also help to improve transition-related outcomes, such as patient satisfaction, and transition readiness, leading to increased quality of healthcare. We assume that this trial is suitable for detecting the multiple beneficial effects of joint visits. Considering ethical issues, participation means a very low risk for patients both in the intervention and control groups.

\section{CONCLUSION}

This RCT will provide a high quality of evidence concerning the value of joint visits compared with the usual transition care in IBD. Our results will help to define to which extent the joint visits can improve individual, IBD-specific and transition-related outcomes.

\section{Author affiliations}

${ }^{1}$ Institute for Translational Medicine, Medical School, University of Pécs, Pécs, Hungary

${ }^{2}$ Heim Pál Children's Hospital, Budapest, Hungary, Budapest, Hungary

${ }^{3}$ Szentágothai Research Centre, Medical School, University of Pécs, Pécs, Hungary

${ }^{4}$ First Department of Pediatrics, University of Debrecen, Hungary, Debrecen, Hungary

${ }^{5}$ Department of Pediatrics, Medical School, University of Pécs, Pécs, Hungary

${ }^{6}$ First Department of Medicine, Medical School, University of Pécs, Pécs, Hungary, Pécs, Hungary

${ }^{7}$ Momentum Gastroenterology Multidisciplinary Research Group, Hungarian Academy of Sciences, University of Szeged, Szeged, Hungary

Contributors All authors read through and made suggestions on the text of the manuscript. The final manuscript was approved by all the authors. AE, GV, PH, ÁV and PS conceptualised the idea of the study. AE, GV, ÁV, PS determined the design of the trial. ZN and DD arranged project administration. The original draft of the manuscript was written by AE, DD and PS. The original draft was reviewed and edited by GV, AT, ATé, PH, ZN, ÁV and NG. The statistical work was performed by NG.

Funding Centre costs (IT, biostatistics, trial organisation, etc) are covered by the project titled 'GINOP-2.3.2-15-2016-00048 - STAY ALIVE' which is cofinanced by the European Union (European Regional Development Fund) within the framework of Programme Széchenyi 2020, by the Human Resources Development Operational Programme Grant, Grant Number: EFOP-3.6.2-16-2017-0006 - LIVE LONGER, which is cofinanced by the European Union (European Regional Development Fund) within the framework of Programme Széchenyi 2020, and by the Grant of the Hungarian Science Foundation (Grant Number: FK 132834). Since no additional treatment is necessary for the study, the general healthcare costs are covered by the National Healthcare System. This study was designed with help of the Centre for Translational Medicine at the University of Pécs. This centre is committed to improve patients' life with research activities (http://www.tm-pte.org/).

Competing interests None declared. Principal investigators of each study sites decline any financial and other competing interests.

Patient and public involvement Patients and/or the public were involved in the design, or conduct, or reporting or dissemination plans of this research. Refer to the Methods section for further details.

Patient consent for publication Not required.

Provenance and peer review Not commissioned; externally peer reviewed.

Open access This is an open access article distributed in accordance with the Creative Commons Attribution Non Commercial (CC BY-NC 4.0) license, which permits others to distribute, remix, adapt, build upon this work non-commercially, and license their derivative works on different terms, provided the original work is properly cited, appropriate credit is given, any changes made indicated, and the use is non-commercial. See: http://creativecommons.org/licenses/by-nc/4.0/.

\section{ORCID iD}

Patrícia Sarlós http://orcid.org/0000-0002-5086-9455

\section{REFERENCES}

1 Van Cleave J, Gortmaker SL, Perrin JM. Dynamics of obesity and chronic health conditions among children and youth. JAMA 2010;303:623-30.

2 Brooks AJ, Smith PJ, Cohen R, et al. Uk guideline on transition of adolescent and young persons with chronic digestive diseases from paediatric to adult care. Gut 2017;66:988-1000.

3 van Rheenen PF, Aloi M, Biron IA, et al. European Crohn's and colitis organisation topical review on transitional care in inflammatory bowel disease. J Crohns Colitis 2017;11:1032-8.

4 Suris J-C, Akre C. Key elements for, and indicators of, a successful transition: an international Delphi study. J Adolesc Health 2015:56:612-8.

5 Fair C, Cuttance J, Sharma N, et al. International and interdisciplinary identification of health care transition outcomes. JAMA Pediatr 2016;170:205-11.

6 van den Brink G, van Gaalen MAC, de Ridder L, et al. Health care transition outcomes in inflammatory bowel disease: a multinational Delphi study. J Crohns Colitis 2019;13:1163-72.

7 Erös A, Soós A, Hegyi P, et al. Spotlight on transition in patients with inflammatory bowel disease: a systematic review. Inflamm Bowel Dis 2020;26:331-46.

8 Cole R, Ashok D, Razack A, et al. Evaluation of outcomes in adolescent inflammatory bowel disease patients following transfer from pediatric to adult health care services: case for transition. $J$ Adolesc Health 2015;57:212-7.

9 Fu N, Jacobson K, Round A, et al. Transition clinic attendance is associated with improved beliefs and attitudes toward medicine in patients with inflammatory bowel disease. World J Gastroenterol 2017;23:5405-11.

10 McCartney S, Lindsay JO, Russell R, et al. Structured transition enhances clinical outcome without an increase in healthcare cost in adolescent patients with IBD: the UK transit study. Gastroenterology 2017;152:S369-S69.

11 Moulton DE, Rosen MJ, Beaulieu DB, et al. Su2015 prospective randomized trial of a progressive pediatric to adult transition of care program in adolescents with inflammatory bowel disease. Gastroenterology 2013;144:S-532-S32.

12 Otto C, Tárnok A, Erős A, et al. Planned transition of adolescent patients with inflammatory bowel disease results in higher remission rates. J Pediatr Nurs 2019;45:62-6. 
13 Romeo E, Ricca O, Angelino G, et al. Transition clinic in inflammatory bowel disease: a two centre model. Journal of Pediatric Gastroenterology and Nutrition 2016;62:338.

14 Yerushalmy-Feler A, Ron Y, Barnea E, et al. Adolescent transition clinic in inflammatory bowel disease: quantitative assessment of selfefficacy skills. Eur J Gastroenterol Hepatol 2017;29:831-7.

15 Alfano G, Nuzio SG, Poeta M, et al. Health care transition of patients with pediatric onset inflammatory bowel disease: a pilot survey. Journal of Pediatric Gastroenterology and Nutrition 2016;62:347

16 Blazquez ML, Hernani MT, Calatayud GA, et al. Transition care program of adolescents with inflammatory bowel disease: perceptions from a survey for patients.. Inflamm Bowel Dis 2018.

17 Williams E, Coates M, Tinsley A, et al. Formalized IBD transition clinic leads to high retention and low no show rates of young adults in a single tertiary center. Am J Gastroenterol 2017;112:S388-9.

18 Schmidt S, Herrmann-Garitz C, Bomba F, et al. A multicenter prospective quasi-experimental study on the impact of a transitionoriented generic patient education program on health service participation and quality of life in adolescents and young adults. Patient Educ Couns 2016;99:421-8.

19 Schmidt S, Markwart H, Bomba F, et al. Differential effect of a patient-education transition intervention in adolescents with IBD vs. diabetes. Eur J Pediatr 2018;177:497-505.

20 Boamah LM, Bohren JR, Pentiuk S, et al. Development and testing of a CD-ROM program for improving adolescent knowledge of inflammatory bowel disease. J Pediatr Gastroenterol Nutr 2010;50:1-5.

21 KKH V, Zhang Y, Denson LA, et al. Evaluation of a novel educational tool in adolescents with inflammatory bowel disease: the neat study. Journal of Pediatric Gastroenterology and Nutrition 2016;63:S66.

22 Zhu X-Q, Li R-X, Chen F. Impact of transitional care on selfmanagement ability and quality of life in patients with inflammatory bowel disease. World Chinese Journal of Digestology 2016;24:3723.

23 Bennett AL, Moore D, Bampton PA, et al. Outcomes and patients' perspectives of transition from paediatric to adult care in inflammatory bowel disease. World J Gastroenterol 2016;22:2611-20.

24 Márta K, Szabó AN, Pécsi D, et al. High versus low energy administration in the early phase of acute pancreatitis (GOULASH trial): protocol of a multicentre randomised double-blind clinical trial. BMJ Open 2017;7:e015874.

25 Kucserik LP, Márta K, Vincze Áron, et al. Endoscopic sphincterotoMy for delaylng choLecystectomy in mild acute biliarY pancreatitis (EMILY study): protocol of a multicentre randomised clinical trial. BMJ Open 2019;9:e025551.

26 Zádori N, Gede N, Antal J, et al. Early elimination of fatty acids iN hypertriglyceridemia-induced acuTe pancreatitis (ELEFANT trial): protocol of an open-label, multicenter, adaptive randomized clinical trial. Pancreatology 2020;20:369-76.

27 Chan A-W, Tetzlaff JM, Altman DG, et al. Spirit 2013 statement: defining standard protocol items for clinical trials. Ann Intern Med 2013;158:200-7.

28 Levine A, Koletzko S, Turner D, et al. ESPGHAN revised Porto criteria for the diagnosis of inflammatory bowel disease in children and adolescents. J Pediatr Gastroenterol Nutr 2014;58:795-806.

29 Levine A, Griffiths A, Markowitz J, et al. Pediatric modification of the Montreal classification for inflammatory bowel disease: the Paris classification. Inflamm Bowel Dis 2011;17:1314-21.

30 Hyams JS, Ferry GD, Mandel FS, et al. Development and validation of a pediatric Crohn's disease activity index. J Pediatr Gastroenterol Nutr 1991;12:439-47.

31 Turner D, Otley AR, Mack D, et al. Development, validation, and evaluation of a pediatric ulcerative colitis activity index: a prospective multicenter study. Gastroenterology 2007;133:423-32.

32 Best WR, Becktel JM, Singleton JW, et al. Development of a Crohn's disease activity index. National cooperative Crohn's disease study. Gastroenterology 1976;70:439-44.

33 Lewis JD, Chuai S, Nessel L, et al. Use of the noninvasive components of the Mayo score to assess clinical response in ulcerative colitis. Inflamm Bowel Dis 2008;14:1660-6.

34 Turner D, Ruemmele FM, Orlanski-Meyer E, et al. Management of paediatric ulcerative colitis, part 1: ambulatory Care-An evidencebased guideline from European Crohn's and colitis organization and European Society of paediatric gastroenterology, hepatology and nutrition. J Pediatr Gastroenterol Nutr 2018;67:257-91.
35 Turner D, Ruemmele FM, Orlanski-Meyer E, et al. Management of paediatric ulcerative colitis, part 2: acute severe Colitis-An evidence-based consensus guideline from the European Crohn's and colitis organization and the European Society of paediatric gastroenterology, hepatology and nutrition. J Pediatr Gastroenterol Nutr 2018;67:292-310.

36 Ruemmele FM, Veres G, Kolho KL, et al. Consensus guidelines of ECCO/ESPGHAN on the medical management of pediatric Crohn's disease. J Crohns Colitis 2014;8:1179-207.

37 Clarke T, Lusher J. Transitioning patients with inflammatory bowel disease (IBD) from adolescent to adult services: a systematic review. Frontline Gastroenterol 2016;7:264-70.

38 Szabó D, Kökönyei G, Arató A, et al. Autoregressive cross-lagged models of IMPACT-III and pediatric Crohn's disease activity indexes during one year infliximab therapy in pediatric patients with Crohn's disease. J Crohns Colitis 2014;8:747-55.

39 Landolt MA, Valsangiacomo Buechel ER, Latal B. Health-Related quality of life in children and adolescents after open-heart surgery. $J$ Pediatr 2008;152:349-55.

40 Otley A, Smith C, Nicholas D, et al. The impact questionnaire: a valid measure of health-related quality of life in pediatric inflammatory bowel disease. J Pediatr Gastroenterol Nutr 2002;35:557-63.

41 Pikarsky AJ, Gervaz P, Wexner SD. Perianal Crohn disease: a new scoring system to evaluate and predict outcome of surgical intervention. Arch Surg 2002;137:774-7.

42 SOLE Study Group. Translation and initial validation of the medication adherence report scale (MARs) in Italian patients with Crohn's disease. Dig Liver Dis 2019;51:640-7.

43 Mahler C, Hermann K, Horne R, et al. Assessing reported adherence to pharmacological treatment recommendations. translation and evaluation of the medication adherence report scale (MARs) in Germany. J Eval Clin Pract 2010;16:574-9.

44 Chan AHY, Horne R, Hankins M, et al. The medication adherence report scale: a measurement tool for eliciting patients' reports of nonadherence. Br J Clin Pharmacol2019.

45 Horne R, Weinman J. Patients' beliefs about prescribed medicines and their role in adherence to treatment in chronic physical illness. $J$ Psychosom Res 1999;47:555-67.

46 Izaguirre MR, Keefer L. Development of a self-efficacy scale for adolescents and young adults with inflammatory bowel disease. $J$ Pediatr Gastroenterol Nutr 2014;59:29-32.

47 Carlsen K, Haddad N, Gordon J, et al. Self-Efficacy and resilience are useful predictors of transition readiness scores in adolescents with inflammatory bowel diseases. Inflamm Bowel Dis 2017;23:341-6.

48 Izaguirre MR, Taft T, Keefer L. Validation of a self-efficacy scale for adolescents and young adults with inflammatory bowel disease. $J$ Pediatr Gastroenterol Nutr 2017;65:546-50.

49 Ferris M, Cohen S, Haberman C, et al. Self-Management and transition readiness assessment: development, reliability, and factor structure of the STARx questionnaire. J Pediatr Nurs 2015;30:691-9.

50 Anelli CG, Len CA, Terreri MTRA, et al. Translation and validation of the transition readiness assessment questionnaire (TRAQ). J Pediatr 2019;95:180-7.

51 Sawicki GS, Lukens-Bull K, Yin X, et al. Measuring the transition readiness of youth with special healthcare needs: validation of the TRAQ--Transition Readiness Assessment Questionnaire. J Pediatr Psychol 2011;36:160-71.

52 Nazareth M, Hart L, Ferris M, et al. A parental report of youth transition readiness: the parent STARx questionnaire (STARx-P) and re-evaluation of the STARx child report. $J$ Pediatr Nurs 2018;38:122-6.

53 Casellas F, Ginard D, Vera I, et al. Development and testing of a new instrument to measure patient satisfaction with health care in inflammatory bowel disease: the Cache questionnaire. Inflamm Bowel Dis 2013;19:559-68.

54 Cohen SA, Aloi M, Arumugam R, et al. Enteric-Coated budesonide for the induction and maintenance of remission of Crohn's disease in children. Curr Med Res Opin 2017;33:1261-8.

55 World Medical Association. World Medical association Declaration of Helsinki: ethical principles for medical research involving human subjects. JAMA 2013;310:2191-4.

56 Dixon JR. The International Conference on harmonization good clinical practice guideline. Quality Assurance 1999;6:65-74. 